\title{
A COMPREHENSIVE REVIEW ON NIOSOMES
}

\section{Preeti Budhlakoti ${ }^{*}$, Kapil Kumar, Deepak Teotia}

Department of Pharmaceutics, Global Institute of Pharmaceutical Education \& Research, Kashipur-244713, Uttarakhand, India

\section{Article Info: Received 25 August 2020; Accepted 20 September. 2020}

DOI: https://doi.org/10.32553/jbpr.v9i5.803

Corresponding author: Preeti Budhlakoti

Conflict of interest statement: No conflict of interest

\section{ABSTRACT:}

Niosomes are a bilayered non-toxic, non-immunogenic nanoparticular delivery system; it is being used for drug delivery. Owing to its cost-effectiveness and easy manufacturing processing, niosomes are gaining popularity as a coveted drug carrier. Main constituents of a niosomes are cholesterol and a non-ionic surfactant, although several additives like charged molecule (Solulan C24) can be added to modify the surface charge of the particle and enhance its stability. There are various methods of manufacturing niosomes like hand shaking method, micro fluidization, sonication, bubble method to name a few. The fact that niosomes are amphiphillic molecules make them a versatile drug carrier as they can entrap both hydrophilic and lipophillic drugs. Applications of niosomes in the pharmaceutical industry therapeutic use, some of the most important ones being as cosmoceuticals, etc. In this review an effort has been made to document the key concepts relating to niosomes and the advances that have been made in terms of research in the field of niosomal drug delivery.

Keywords: niosomes, nanoparticles, cholesterol, carrier, non-ionic, vesicles.

\section{INTRODUCTION}

Niosomes are vesicles, which serve as novel drug delivery systems. Niosomes are non-ionic surfactant based unilamellar or multilamellar vesicles depending on the methods of preparation. The bilayer is made of phospholipid and they can be used as carriers of amphiphilic and lipophilic drugs. The niosomes are made of a surfactant bilayer with its hydrophilic ends exposed on the outside and inside of the vesicle while the hydrophobic chains face each other within the bilayer ${ }^{1}$. Hence, the vesicle entraps the hydrophilic drugs within the space enclosed in the vesicle while the hydrophobic drugs are embedded within the bilayer itself ${ }^{2}$.

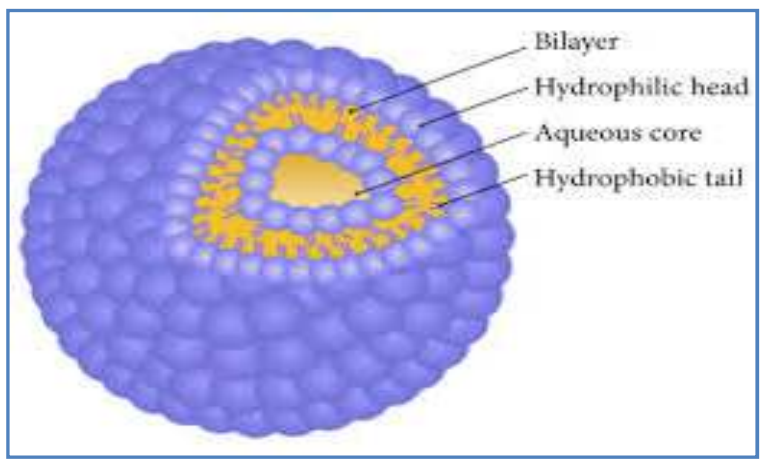

Figure 1: Niosomes

Niosomes are microscopic in size and their size lies in nanometer scale. They can be formulated by different methods like, hand shaking, ether Injection, bubble method, sonication, micro fluidization ,reverse phase evaporation technique and trans membrane $\mathrm{pH}$ gradient. The formulated Niosomes are evaluated by size, shape and morphology entrapment efficiency, invitro release, and stability studies ${ }^{3}$.

\section{Composition of Niosomes}

They are consisted of two essential components like cholesterol and non ionic surfactants.

\section{Cholesterol}

Cholesterol is a natural steroid found in the cell membranes. Cholesterol is used to provide rigidity and proper shape, conformation to the niosomes preparations. It forms the vesicles with non ionic surfactants, reduces agglomeration and provides greater stability. Cholesterol is also known to prevent gel to liquid phase transition of niosomal systems. As a result of this the noisome become less leaky in nature ${ }^{4}$.

\section{Non-lonic Surfactants}

Non-ionic surfactants act as main component in Niosome formulations. Generally, they possess hydrophilic head group and a hydrophobic tail and possess high interfacial activity. The hydrophobic moiety may consist of $1 / 2 / 3$ alkyl chains or per fluro group or in certain cases a single stearyl group ${ }^{5}$. 
Surfactants of non ionic groups like spans are available in different grades like span 20 , span 40 , span 60 , span 80 and span 85 . Similarly the surfactant of Tweens also has different grades such as Tween 20, Tween 40, Tween 60 and tween 80 are generally used for the preparation of niosomes ${ }^{6}$.

\section{Advantages of Niosomes}

- Reduced dose is required to achieve the desired effects.

- The vesicles can release the drug in a controlled manner.

- They are osmotically active and stable; as a result the stability of entrapped drug gets increased.

- Handling and storage of surfactants in noisome requires no special conditions.

- Subsequent decrease in side effect ${ }^{7}$.

- They can be made to reach the site of action by oral, parenteral as well as topical routes.

- Niosomes are both hydrophilic and lipophilic in nature and can accommodate a large number of drugs with a wide range of solubilities ${ }^{8}$.

\section{Disadvantages of Niosomes}

- The aqueous suspension of niosomes may limited shelf life

- Due to fusion, aggregation, leakage of entrapped drug and hydrolysis of encapsulated drug.

- The methods of preparation of multilamellar vesicles such as sonication are time consuming and may require specilazed equipment for processing ${ }^{9}$.

- It is a high production cost.

- Leaking of entrapped drug.

METHODS OF PREPARATION

Hand Shaking Method (Thin Film Hydration Technique)

Surfactant and cholesterol are dissolved in a volatile organic solvent (diethyl ether chloroform or methanol) in a round bottom flask. The organic solvent is removed under vacuum at room temperature $\left(20^{\circ} \mathrm{C}\right)$ using rotary evaporator leaving a thin layer of solid mixture deposited on the wall of the flask. The dried surfactant film can be rehydrated with aqueous phase at temperature with gentle agitation. This process forms typical multilamellar niosomes ${ }^{10}$.

\section{Preparation Step:}

Step- 1 Surfactant + cholesterol I+ solvent

Step- 2 Remove organic solvent at room temperature.

Step- 3 Thin layer of solid mixture formed on the walls of flask

Step- 4 Film can be rehydrated to form multilamellar niosomes ${ }^{11}$.

\section{Micro Fluidization}

Micro fluidization is a recent technique used to prepare unilamellar vesicles of defined size distribution. A microfludizer is used to pump the fluid at a very high pressure $(10,000 \mathrm{psi})$ through a $5 \mathrm{pm}$ screen. It is then forced along defined micro channels, which direct two streams of fluid to collide together at right angles, thereby affecting a very efficient transfer of energy. The lipid/surfactants can be introduced into the fluidizer .The fluid collected can be recycled until spherical vesicles are obtained. Uniform and small vesicles are obtained $^{12}$.

Preparation Step:

Step- 1 Two ultra high speed jets inside interaction chamber

Step- 2 Impingement of thin layer of liquid in micro channels

Step- 3 Formation of uniform niosomes

Reverse Phase Evaporation Technique

Creation of a solution of cholesterol and surfactant (1:1) in a mixture of ether and chloroform. An aqueous phase containing drug to be added to this and the resulting two phases are sonicated at $4-5^{\circ} \mathrm{C}$. A clear gel formed which is further sonicated after the addition of phosphate buffered saline (PBS). The organic phase is removed at $40^{\circ} \mathrm{C}$ under low pressure. The resulting viscous niosome suspension is formed which can be diluted with PBS and heated on a water bath at $60^{\circ} \mathrm{C}$ for $10 \mathrm{~min}$ to yield niosomes. The preparation of tropicamide niosomes using Tween 85 by this method ${ }^{13}$.

Preparation Step:

Step- 1 Cholesterol + surfactant dissolved in ether + chloroform

Step- 2 Drug in aqueous phase is added to above mixture

Step- 3 Sonicated at $5^{\circ} \mathrm{C}$ and again sonicated after adding PBS

Step- 4 Viscous niosomes suspension is diluted with PBS

Step- 5 Organic phase is removed at $40^{\circ} \mathrm{C}$ at low pressure

Step- 6 Heated on a water bath for $60^{\circ} \mathrm{C}$ for 10 mints to yield niosomes ${ }^{14}$.

Ether Injection Method

Introduction of mixture of surfactant and cholesterol dissolved in diethyl ether into warm water bath maintained at $60^{\circ} \mathrm{C}$. The surfactant mixture in ether is injected into an aqueous phase using a 14-gauze needle syringe. Vaporization of ether leads to formation of single layered vesicles. Depending upon the conditions used the diameter of the vesicle range from 50 to1000 $\mathrm{nm}^{15}$.

Preparation Steps

Step- 1 Surfactant and cholesterol is dissolved in diethyl ether

Step- 2 Then injected warm water maintained at $60^{\circ} \mathrm{C}$ through a 14 gauze needle syringe

Step- 3 Ether is vaporized to form single layered niosomes

Trans Membrane Ph Gradient

Surfactant and cholesterol are dissolved in chloroform. The solvent is then evaporated under reduced pressure to get a thin film on the wall of the round bottom flask. 
The film is hydrated with citric acid (pH4.0) by vortex mixing. The multilamellar vesicles are frozen and shared 3 times and later sonicated. To this niosomal suspension, aqueous solution containing $10 \mathrm{mg} / \mathrm{ml}$ of drug is added and vortex. The $\mathrm{pH}$ of the sample is then raised to 7.0-7.2 with $1 \mathrm{M}$ disodium phosphate. This mixture is later heated at $60^{\circ} \mathrm{C}$ for 10 minutes to give niosomes ${ }^{16}$.

\section{Preparation Steps:}

Step- 1 Surfactant + cholesterol in chloroform

Step- 2 Solvent is evaporated under reduced pressure

Step- 3 Thin film is deposited on the walls of RBF

Step- 4 Hydrated with citric acid (PH 4.0) by vortex mixing

Step- 53 cycles of freezing and shared then sonication Step- 6 Addition of aqueous drug solution and vortoxing Step- $7 \mathrm{pH}$ raised to $7.0-7.2$ by $1 \mathrm{M}$ disodium phosphate Step- 8 Mixture is heated at $60^{\circ} \mathrm{C}$ for 10 minutes to give niosomes.

\section{The "Bubble" Method}

A recently developed technique which allows the preparation of niosomes without the use of organic solvent. . The bubbling unit consists of round-bottomed flask with three necks, and this is positioned in water bath to control the temperature. Water-cooled reflux and thermometer is positioned in the first and second neck, while the third neck is used to supply nitrogen. Cholesterol and surfactant are dispersed together in a buffer $(\mathrm{pH} 7.4)$ at $70^{\circ} \mathrm{C}$; this dispersion is mixed for a period of 15 seconds with high shear homogenizer and immediately afterwards, "bubbled" at $70^{\circ} \mathrm{C}$ using nitrogen gas to yield niosomes ${ }^{17}$.

Preparation Steps:

Step- 1 The preparation of niosomes without the use of organic solvent

Step- 2 RBF as bubbling unit with three necks in water bath

Step- 3 Water cooled reflux, thermometer and nitrogen supply by three necks

Step- 4 Cholesterol + surfactant dispersed in buffer $\mathrm{pH}$ 7.4 at $70^{\circ} \mathrm{C}$

Step- 5 Above dispersion is homogenized for $15 \mathrm{sec}$ and then bubbled with nitrogen gas at $70^{\circ} \mathrm{C}$

Step- 6 To get niosomes.

\section{Sonication}

A typical method of production of the vesicles is by sonication of solution. In this method an aliquot of drug solution in buffer is added to the surfactant/ cholesterol mixture in a $10-\mathrm{ml}$ glass vial. The mixture is probe sonicated at $60^{\circ} \mathrm{C}$ for 3 minutes using a sonicator with a titanium probe to yield niosomes.

\section{Preparation Steps}

Step-1 Drug buffer+ surfactant/cholesterol in $10 \mathrm{ml}$ in glass vial

Step- 2 Above mixture is sonicated for 3 mins . At $60^{\circ} \mathrm{C}$ using titanium probe yield niosomes.

\section{EVALUATION PARAMETERS}

- Size, Shape And Morphology:

Freeze Fracture Electron Microscopy: Visualize the vesicular structure of surfactant based vesicles.

Photon Correlation Spectroscopy: Determine mean diameter of the vesicles.

Electron Microscopy: Morphological studies of vesicles.

\section{- Entrapment Efficiency:}

The entrapment efficiency of ethosomes can be measured by the ultracentrifugation technique.

Entrapment efficiency $(\%)=($ Amount of drug addedTotal amount of drug) $\times 10$

\section{- Vesicle Surface Charge:}

Determined by measurement of electrophoretic mobility and expressed in terms of zeta potential ${ }^{18}$.

\section{- In-Vitro Release:}

A method of in-vitro release rate study includes the use of dialysis tubing. A dialysis sac is washed and soaked in distilled water. The vesicle suspension is pipette into a bag made up of the tubing and sealed. The bag containing the vesicle is placed in $200 \mathrm{ml}$ of buffer solution in a $250 \mathrm{ml}$ beaker with constant shaking at $25^{\circ} \mathrm{C}$ and $37^{\circ} \mathrm{C}$.At various time intervals; the buffer is analyzed for the drug content by an appropriate assay method of vesicles during the cycle.

\section{- Stability Studies}

The stability of vesicles can be determined by assessing the size and structure of the vesicles over time. Mean size is measured by dynamic light scattering (DLS) and structure changes are observed by transmission electron microscopy (TEM) ${ }^{19}$.

\section{Applications:}

The applications can be mainly classified into three categories.

\section{For Controlled Release of Drugs:}

- Ophthalmic Drug Delivery.

2. To Improve the Stability and Physical Properties of the Drugs :

- To Increase Oral Bioavailability.

- For Improvement of Stability of Peptide Drugs.

- To Promote Trans dermal Delivery of Drugs.

- Improvement of Stability of Immunological Products.

- Improve Anti-inflammatory Activity.

3. For Targeting and Retention of Drug in Blood Circulation :

- For Liver Targeting.

- To Improve the Efficacy of Drugs in Cancer Therapy. 
- In Diagnostic Imaging.

- Carrier for Hemoglobin.

1. For Controlled Release :

The release rate of drugs like withaferin and gliclazide from the niosomes was found slower as compared to other dosage forms ${ }^{20}$.

\section{In Ophthalmic Drug Delivery:}

Experimental results of the water soluble antibiotic Gentamicin sulphate showed a substantial change in the release rate. Beside this, the percent entrapment efficiency of gentamicin sulphate was altered when administered as niosomes. Also, as compared to normal drug solution, niosomes of drug show slow release.

Niosomal formulation containing timolol maleate $(0.25 \%)$ prepared by chitosan coating exhibited more effect on intra ocular tension with fewer side effects as compared to the marketed formulation ${ }^{7}$.

\section{To Improve Drugs Stability and Physical Properties:}

\section{To Increase Oral Bioavailability:}

With the formulation of niosomes, the oral bioavailability of the Acyclovir as well as Griseofulvin was increased as compared to the drug alone.

For Improvement of Stability of Peptide Drugs:

8-arginin vasopressin, 9-glycinamide- $\omega$

The in vitro release of insulin from niosomes formulated by span 40 and span 60 in simulated intestinal fluid was lower than the niosomes formulated by span 20 and span 80.

Niosomes prepared by the span 60 has high resistance against proteolytic enzyme and exhibit good stability in storage temperature and in presence of sodium deoxycholate ${ }^{8}$.

To Promote Tran Dermal Delivery of Drugs:

Many drugs such as lidocaine, estradiol, cyclosporine etc. are used for topical and transdermal drug delivery system by formulating them as niosomes.

The niosomes of natural compound, ammonium glycyrrhizinate were formulated for effective antiinflammatory activity using new non-ionic surfactant, bola surfactant-span 80-cholesterol (2: 3: 1 ratio).

Experimental study showed that the bola niosomes were able to promote the intracellular uptake of ammonium glycerrhizinic acid ${ }^{11}$.

Improvement of Stability of Immunological Products:

Important tool for immunological selectivity, low toxicity and more stability of the incorporated active moiety.

Niosomes are better stimulant of IgG2.

To Improve Anti-inflammatory Activity:

Niosomal formulation of diclofenac sodium prepared with $70 \%$ cholesterol showed greater anti-inflammatory effect as compared to the free drug.

Similarly, nimesulide and flurbiprofen showed greater activity than the free drug $^{12}$.
3. For Targeting and Retention of Drug in Blood Circulation:

For Liver Targeting:

Methotrexate was reported to be selectively taken up by liver cells after administration as a niosomal drug delivery system.

To Improve the Efficacy of Drugs in Cancer Therapy:

Niosomes of doxorubicin prepared from C16 monoalkyl glycerol ether with or without cholesterol, exhibited an increased level of doxorubicin in tumor cells, serum and lungs, but not in liver and spleen.

Niosomal preparation of methotrexate exhibited greater antitumor activity \& low side effects as compared to plain drug solution ${ }^{8}$

In Diagnostic Imaging:

It has been studied that niosomes can also act as a carrier radiopharmaceuticals and showed site specificity for spleen and liver for their imaging studies using 99mTc labelled DTPA (diethylene triamine pentaacetic acid) containing niosomes.

Conjugated niosomal formulations of gadobenate with (Npalmitoyl-glucosamine, NPG), PEG 4400 and both PEG and NPG can be used to increased tumor targeting of a paramagnetic agent. 3.5 Carrier for Haemoglobin:

Carrier for Haemoglobin:

Haemoglobin loaded niosomes prepared by reverse phase evaporation method is permeable to oxygen.

Usefulness of Niosomes in Cosmetics:

Niosomes of $\mathrm{N}$-acetyl glucosamine are prepared due to its potential in the delivery of hydrophilic and hydrophobic drugs in topical form and improved penetration into the skin.

Prepared formulations improved the extent of drug localized in the skin, as needed in hyper pigmentation disorders. Elastic niosomes showed increased permeation through the skin which will be beneficial for topical anti-aging application. Niosomes are suitable for skin moisturising and tanning products Niosomes were prepared as possible approach to improve the low skin penetration and bioavailability shown by conventional topical vehicle for minoxidil ${ }^{17,20}$.

\section{CONCLUSION}

Niosomes provide incorporating the drug into for a better targeting of the drug at appropriate tissue destination. They present a structure similar to liposome and hence they can represent alternative vesicular systems with respect to liposomes. Candidates drug delivery as compared to liposome due to various factors like cost, stability etc.various type of drug deliveries can be possible using niosomes like targeting, ophthalmic, topical, parental etc.

REFERENCES 
1. Madhav Nvs* and Saini A, Niosomes: A Novel Drug Delivery System, International Journal Of Research In Pharmacy And Chemistry, 2011, 1(3), 498-511.

2. Lohumi Ashutosh, Rawat Suman, A Novel Drug Delivery System: Niosomes Review, Journal of Drug Delivery \& Therapeutics; 2012, 2(5), 129-135.

3. Bhartee Pathak, Kapil Kumar. Buccal drug delivery system: a tool for the effective delivery of pharmaceuticals. Universal Journal of Pharmaceutical Research 2017; 2(3): 19-24.

4. Pawar Sd *, Pawar Rg, Niosome: An Unique Drug Delivery System, International journal Of Pharmacy, Biology and Allied Sciences, April, 2012, 1(3): 406416.

5. Umar S, Onyekachi MK. Development and evaluation of transdermal gel of Lornoxicam. Universal Journal of Pharmaceutical Research 2017; 2(1): 15-18.

6. Rajesh Z. Mujoriya, Niosomal Drug Delivery System - A Review, International Journal Of Applied Pharmaceutics, Vol 3, Issue 3, 2011,7-10.

7. Kaur G, Paliwal S. Formulation and evaluation of etoricoxib microbeads for sustained drug delivery. Universal Journal of Pharmaceutical Research 2019; 4(1): 35-39.

8. Textbook of industrial pharmacy, drug delivery system \& cosmetic and herbal drug technology. SHOBHA RANI. RH. 2014, universities press.

9. Elsaied EH, Dawaba HM, Ibrahim EA, Afouna MI. Investigation of proniosomes gel as a promising carrier for transdermal delivery of Glimepiride. Universal Journal of Pharmaceutical Research 2016; 1(2): 1-10.

10. Introduction to novel drug delivery system;N.K.JAIN.2017s, Vallabhprakashan
11. Nweje-Anyalowu Paul C, Anyalogbu Ernest $A A$, White Alalibo Jim. Design and evaluation of chronotherapeutic pulsatile drug delivery system of Cilnidipine. Universal Journal of Pharmaceutical Research 2017; 2(5): 15-18.

12. Chigbo UJ, Ugochukwu AE, John DF. Dendrimers: a novel tool for drug delivery and targeting. Universal Journal of Pharmaceutical Research 2017; 2(3): 3440.

13. Controlled and novel drug delivery; N.K.JAIN. 2017, CBS publishers.

14. Vyas S. P. And Khar R. K., Targeted and Controlled Drug Delivery System, 1stEdition, 2002, CBS Publication; 249 - 277.

15. Peter OI, Ifeoma UC. Development and evaluation of Albendazole microcapsule for colonic drug delivery system. Universal Journal of Pharmaceutical Research 2017; 2(2): 4-7.

16. Chioma ED. Formulation and evaluation of etodolac niosomes by modified ether injection technique. Universal Journal of Pharmaceutical Research 2016 1(1): 1-4.

17. Jain N. K., Controlled and novel Drug Delivery, 1st edition 2001, CBS Publication; 292 - 301.

18. Chauhan N, Kumar K, Pant NC. An updated review on transfersomes: a novel vesicular system for transdermal drug delivery. Universal Journal of Pharmaceutical Research 2017; 2(4): 42-45.

19. Arora Rajnish and Jain C.P., Advances in niosomes as a drug carrier: A review, Asian journal of pharmaceutics, Vol-1, Issue1, April-june 2007, 2939.

20. Ugochukwu $A E$, Nnedimkpa OJ, Rita NO. Preparation and characterization of Tolterodine tartrate proniosomes, Universal Journal of Pharmaceutical Research 2017; 2(2): 1-3. 